\title{
Improved Traffic Lane Edge Detection Algorithm Based on Mathematical Morphology
}

\author{
Liying $\mathrm{Wu}^{1, \mathrm{a}}$, Qiang $\mathrm{Yu}{ }^{1, \mathrm{~b}}$,Tongqiang $\mathrm{Xu}{ }^{2}$ and Shuo Zhang ${ }^{1}$ \\ ${ }^{1}$ School of Automobile, Chang'an University, Xi'an 710064,China \\ ${ }^{2}$ ShandongLinyi Engineering Machinery St,Linyi,276023, China \\ a2843261391@qq.com, ${ }^{\mathrm{b}} 2399053899 @ q q . c o m$
}

Keywords:Lane edge detection; morphological filter; Otsu; morphology.

\begin{abstract}
In view of the limited anti-noise of traditional traffic lane edge detection algorithm, the paper put forward to a new traffic lane edge detection algorithm based on traditional mathematical morphology. For solving the problem of uneven road surface caused by illumination and rainy, this paper combine two method of morphological filter and Otsu to segment image and take the improved algorithm to detect the traffic lane edge. Finally, through collecting the images of a campus road under different environment factors, detect traffic lane edge by using the improved algorithm .The simulation results show that, under various weather conditions, this algorithm can detect traffic lane edge correctly and continuously, and have a strong capability of anti-noise. This improved algorithm is of certain guiding significance in practice.
\end{abstract}

\section{Introduction}

With the rapid growth of car ownership, traffic accident rate has been greatly increased, which has brought huge economic losses and casualties. Improper operation of the driver in the driving process including exceed speed, not to give way, illegal road occupation, converse running, fatigue driving and others are the main reason to cause traffic accidents. In this background, unmanned-drive vehicle became an issue. It integrates machine vision, artificial intelligence, information processing, wireless communications and other high-tech in one. Among them, the visual acquisition system is the key technology of the normal driving of intelligent vehicles and in the visual acquisition system, the edge detection is very important.

In recent years, many different algorithms including differential operator, LOG operator and Canny operator and others have been applied to the edge detection Although these algorithms have some advantages, there are also some limitations in lane line detection. The edge processed by these algorithms is fuzzy or produce more false edges, which cause some trouble for next lane line extraction. Mathematical morphology is a new method for image processing and pattern recognition, it designs a set of operations, concepts and algorithms to describe the basic features of the image and its anti noise ability is better than the differential algorithm, and the edge of the extraction is relatively smooth. But the environment faced by unmanned-drive vehicle is more complex. In the environment of strong illumination, rainy weather and others, the traditional mathematical morphology edge detection algorithm cannot meet the requirements of lane edge detection.

In order to solve these problems mentioned above, this paper proposes an improved algorithm for lane line detection. The image is filtered by morphological filter firstly and segmented by Otsu secondly. Finally, this paper adapt to the edge detection operator that combined different directions of structural elements to detect the lane edge of the road. This method has strong anti noise ability and the extracted edge of the lane is clear and the false edges are also greatly reduced.

\section{Lane Edge detection algorithm based on morphological}

\subsection{Traditional Lane Line Edge Detection Algorithm Based on Morphological}

Morphology edge detection operator is a new image processing and analysis technology. The rule are expansion, corrosion, open operation and close operation. 
Traditional lane edge detection algorithms based on morphological are put forward on these operations including three types of expansion, corrosion and dilate-erode operator.

(1) Expansion operation: $E_{d}(\mathrm{x}, \mathrm{y})=(I \oplus B)(x, y)-I(x, y)$

(2) Corrosion operation: $E_{e}(\mathrm{x}, \mathrm{y})=I(x, y)-(I \Theta B)(x, y)$

(3) Dilate-erode operator: $E_{d e}(\mathrm{x}, \mathrm{y})=(I \oplus B)(x, y)-(I \Theta B)(x, y)$

The image edge processed by expansion operation will be blurred. The image edge processed by corrosion operation will lose some details. In order to get the ideal edge, based on the edge detection algorithm, reference 7 defines a new edge detection algorithm as follows:

$E(\mathrm{x}, \mathrm{y})=E_{d}(\mathrm{x}, \mathrm{y})+0.5 E_{d e c}(\mathrm{x}, \mathrm{y})$

Of which:

$\left\{\begin{array}{l}E_{d e c}(\mathrm{x}, \mathrm{y})=E_{\max }(\mathrm{x}, \mathrm{y})-E_{\min }(\mathrm{x}, \mathrm{y}) \\ E_{\max }(\mathrm{x}, \mathrm{y})=\max \left\{E_{d}(\mathrm{x}, \mathrm{y}), E_{e}(\mathrm{x}, \mathrm{y}), E_{d e}(\mathrm{x}, \mathrm{y})\right\} \\ E_{\min }(\mathrm{x}, \mathrm{y})=\min \left\{E_{d}(\mathrm{x}, \mathrm{y}), E_{e}(\mathrm{x}, \mathrm{y}), E_{d e}(\mathrm{x}, \mathrm{y})\right\}\end{array}\right.$

The algorithm mentioned in the reference 7 reduces the ambiguity of the image by superimpose part of the edge details of original image. But these four edge detection are used by a single structure element, only contains one kind of geometric information, therefore, the strain capacity to complex environment is poor.

\subsection{Improved Lane Line Edge Detection Algorithm Based on Morphological}

In order to solve the problem that the single structure element cannot maintain the image edge details and the poor ability to resist noise, the edge detection operator of multi - scale and multi structure elements is given by reference 8 , mathematical definitions are as follow:

$$
\left\{\begin{array}{l}
E(\mathrm{x}, \mathrm{y})=0.5 E_{M}(\mathrm{x}, \mathrm{y})+0.5 E_{N}(\mathrm{x}, \mathrm{y}) \\
E_{M}(\mathrm{x}, \mathrm{y})=\sum_{i=1}^{4} a_{i} E_{i}(\mathrm{x}, \mathrm{y}) \\
E_{N}(\mathrm{x}, \mathrm{y})=\sum_{i=1}^{3} a_{i} E_{i}(\mathrm{x}, \mathrm{y})
\end{array}\right.
$$

Where $a_{i}$ is weighting coefficient, $E_{u}(\mathrm{x}, \mathrm{y})$ is result of multi - structure element edge detection, $E_{N}(\mathrm{x}, \mathrm{y})$ is result of multi - scale element edge detection.

Although the algorithm can keep the image detail information and remove the noise better while detecting the edge of the image, because of its complex operation, long processing time and other reasons, it cannot be applied to the edge detection of lane line detection with high real-time requirements. Aim at the problem, the paper improves the algorithm above based on the shape feature of road image. By using single - scale and four - structure elements to carry out morphological operations on road image, problems of single structure element and the real-time requirements in the detection process are solved. Mathematical definition of improved lane line edge detection algorithm based on morphological is that:

$$
\left.\left.E d g e=\left[I(x, y) \Theta B_{1}\right) \oplus B_{2} \oplus B_{3} \oplus B_{4}\right]-\left[I(x, y) \oplus B_{1}\right) \Theta B_{2} \Theta B_{3} \Theta B_{4}\right](7)
$$

Where $I(x, y)$ is input gray image. $B_{1}, B_{2}, B_{3}, B_{4}$ are respectively four different structural elements. Through test comparison, it is found that the result of edge detection and algorithm adaptability are the best. Of which:

$$
B_{1}=\left[\begin{array}{lll}
1 & 1 & 1 \\
1 & 1 & 1 \\
1 & 1 & 1
\end{array}\right], B_{2}=\left[\begin{array}{lll}
0 & 1 & 0 \\
1 & 1 & 1 \\
0 & 1 & 0
\end{array}\right], B_{3}=\left[\begin{array}{lll}
0 & 0 & 1 \\
0 & 1 & 0 \\
1 & 0 & 0
\end{array}\right], B_{4}=\left[\begin{array}{lll}
1 & 0 & 0 \\
0 & 1 & 0 \\
0 & 0 & 1
\end{array}\right]
$$

$B_{1}, B_{2}$ are square structural element and cross-shaped structural element respectively. $B_{3}, B_{4}$ are the structural element made with $3 \times 3$ scale at the direction of 45 degrees and 135 degrees along the horizontal direction respectively. As can be seen, these four kinds of structural elements almost cover all directions of the road image, it can obtain the complex edge and texture features of road image and have a high ability to anti noise. 


\section{Experiment and Result Analysis}

\subsection{Image Acquisition}

This paper collects the image of a structural road. From the picture, we can see that there are trees on two sides of the road. When the light intensity is strong, it will produce uneven shade and there will be covered by leaves in the rainy weather, which will increase the difficulty of the lane line edge detection.

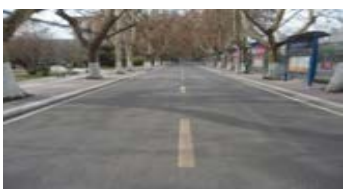

fig2. Structural road

\subsection{The Effect of Morphological Filter}

This paper constructs morphological filter based on morphology. In order to illustrate the effect of the filter, the road image is filtered to remove noise using median filter and morphological filter constructed in this paper respectively. Then use Otsu to segment the filtered image. The contrast effect is shown in figure 3-4.

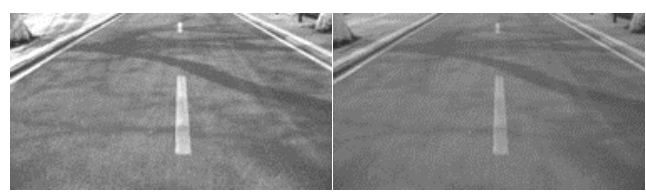

(a) Morphological Filtering (b) Median Filtering fig3. The Contrast of Image enhancement

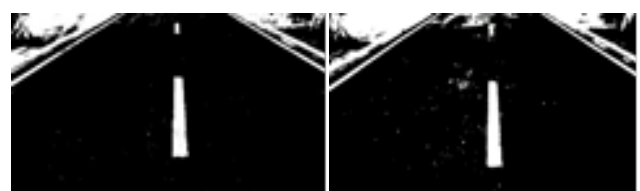

(a) Morphological Filtering (b) Median Filtering

fig4. The Contrast of Image segmentation

From figure 3 we can see that the morphological filter constructed in this paper makes the contrast of road image significantly enhanced. From figure 4 we can see that the ability of it to suppress the noise is better than the median filter.

\subsection{Improved Algorithm for Lane Detection}

In order to illustrate the detection effect of the improved algorithm, canny operator, LOG operator, traditional morphological detection algorithm and detection algorithm of this paper are used to detect the lane edge. The contrast effect is shown in figure 5.
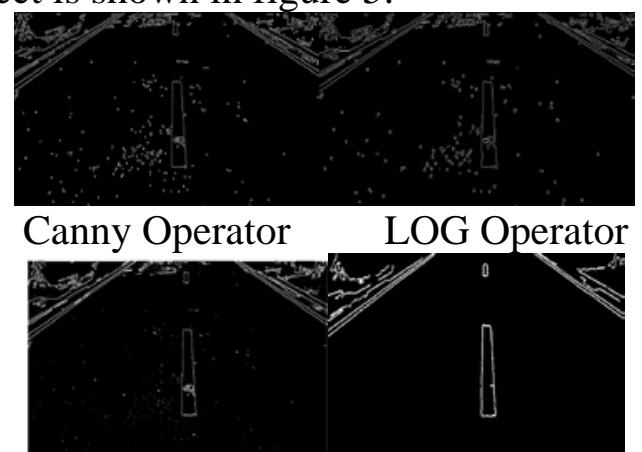

\section{Traditional Detection Algorithm Detection Algorithm of This Paper}

fig5. Lane Edge Detection in Light Weather

From figure 5, we can see that canny operator and LOG operator influenced by noise are very serious and produce more false edge. Although traditional morphological detection algorithm has improved, the anti noise ability is still less than ideal. The lane line edge detected by the new algorithm of this paper is clear and continuous, and the ability of restraining noise is better than the traditional detection algorithm. 


\subsection{The applicability of the improved algorithm other environmental factors}

Through 6.3 we can see that the improved algorithm in this paper is effective in the light environment. Below we collect the structure of campus road images in different environments, use the algorithm for lane detection and observe the applicability of the algorithm. Lane edge detection results are shown in Figure 6-9

(1) Edge Detection of Lane Line under Cloudy Conditions and Covered with Leaves

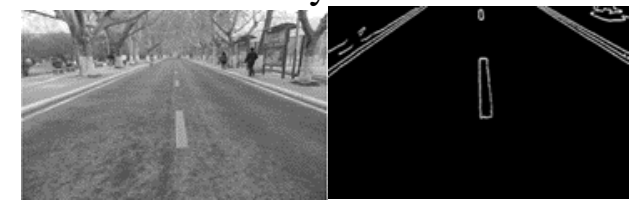

Original Image improved algorithm

fig6. Edge Detection under Cloudy Conditions

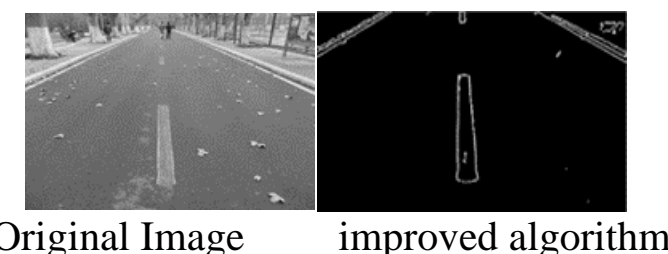

fig7. Edge Detection under Cloudy Conditions Covered With Leaves

(2) Edge Detection of Lane Line under Rainy Conditions and Covered with Leaves

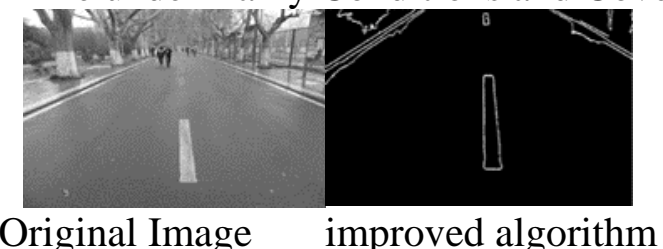

Fig8. Edge Detection under Rainy Conditions

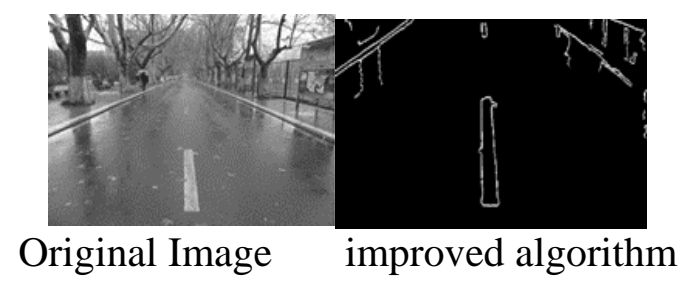

Fig9. Edge Detection under Rainy Conditions Covered With Leaves

From the image we can see that the proposed algorithm can achieve better results under different climate conditions. And when the pavement covered with water and leaves can also detect the lane line edge more accurately, with a strong applicability.

\section{Conclusion}

This paper collects structural campus road images under different weather conditions and segment image above with the constructed morphological filter and Otsu. Finally detect the lane line edge by using the improved algorithm of this paper. The results show that the proposed algorithm can detect the lane line edge clearly and continuously and suppress the noise well under light, rain, covered with leaves and other environmental factors. It is advantageous to the extraction of the lane line,which has a certain practical value. This algorithm has the advantages of high precision, high accuracy and applicability, but it needs to be improved in real time due to the high complexity of the algorithm. 


\section{Acknowledgement}

This work is supported by Key project of National Natural Science Foundation of China: research and development of intelligent test environment for unmanned vehicle (90920305). The corresponding author is Dr. Qiang Yu, Chang’an University, Email: 2399053899@qq.com

\section{References}

[1]Zhang Shuo. Research on Intelligent Vehicle Motion Control in Microscopic Traffic Environment [D].Xi'an: Chang'an university, 2014:1-9.

[2] Carsten STEGER, Markus Ulrich, Christian Wiedemann. Machine Vision Algorithms and Applications [M]. Wiley-VCH, 2008.

[3] Yang Dan, Zhao Haibin. Image Processing Examples with Malab[M].Beijing: Tsinghua University Press ,2013.

[4] Richard SZELISKI. Computer Vision: Algorithms and Applications [M]. Beijing: Tsinghua University Press, 2012.

[5] Rafael C. Gonzalez, Richard E. Woods. Digital Image Processing Third Edition[M]. Beijing: Publishing House Of Electronics Industry, 2005.

[6]Zhang Yujin. Image Engineer [M]. Beijing: Tsinghua University Press, 2005.

[7]Fu Yongqing, Wang Yongsheng. An Algorithm for Edge Detection of Gray-scale Image Based on Mathematical Morphology [J]. Journal of Harbin Engineering University, 2005,26(5):685-687.

[8]Xu Guobao, Wang Ji. Adaptive Algorithm of Edge Detection Based on Mathematical Morphology [J] . Journal of Computer Applications, 2009,29(4):997-1002.

[9]Xu Yan, Lei Tao. Research of Traffic Lane Detection Algorithm Based on Morphology Method [J].Journal of Railway, 2009,31(1):107-110.

[10]Ou Xianfeng, Jia Zhenhong. Research on Edge Detection of Gray Digital Image Based on Multi-structural Anti-noise Dilation-erosion Operator [J] . Laser Journal, 2009,30(1):40-41.

[11] Sun Junxi, Gu Dongbing. A multi-scale edge detection algorithm based on wavelet domain vector hidden markov tree model [J].Pattern Recognition,2004,37(7):1315-1324.

[12]Xiao Mei, Han Chongzhao. Morphologic Edge Detection Based on Multi-scale Contour Structuring Elements [J] .Journal of Xi'an Jiao tong University. 2005,39(6):659-660. 